\title{
Dynamics of Hotspot Formation in Nanostructured Superconducting Stripes Excited with Single Photons
}

\author{
A. Jukna ${ }^{a, b}$, J. Kitaygorsky ${ }^{a}$, D. $\operatorname{Pan}^{a}, \mathrm{~A} \cdot \mathrm{Cross}^{a}$,
} A. Perlman ${ }^{a}$, I. Komissarov ${ }^{a}, \mathrm{O} \cdot \mathrm{Okunev}^{c}, \mathrm{~K} \cdot \mathrm{Smirnov}^{c}$, A. Korneev ${ }^{c}$, G. Chulkova $^{c}$, I. Milostnaya ${ }^{c}$, B. Voronov $^{c}$, G.N. Gol'tsman ${ }^{c}$ And R. Sobolewski ${ }^{a}$

${ }^{a}$ Department of Electrical and Computer Engineering and Laboratory for Laser Energetics, University of Rochester, Rochester NY 14627-0231, USA

${ }^{b}$ High-Tc-Superconductivity Laboratory, Semiconductor Physics Institute

A. Goštauto 11, LT-01108 Vilnius, Lithuania

${ }^{c}$ Department of Physics, Moscow State Pedagogical University

119992 Moscow, Russia

Dynamics of a resistive hotspot formation by near-infrared-wavelength single photons in nanowire-type superconducting NbN stripes was investigated. Numerical simulations of ultrafast thermalization of photon-excited nonequilibrium quasiparticles, their multiplication and out-diffusion from a site of the photon absorption demonstrate that $1.55 \mu \mathrm{m}$ wavelength photons create in an ultrathin, two-dimensional superconducting film a resistive hotspot with the diameter which depends on the photon energy, and the nanowire temperature and biasing conditions. Our hotspot model indicates that under the subcritical current bias of the 2D stripe, the electric field penetrates the superconductor at the hotspot boundary, leading to suppression of the stripe superconducting properties and accelerated development of a voltage transient across the stripe.

PACS numbers: 74.25.Fy, 74.25.Sv, 74.45.+c

\section{Introduction and hotspot model}

The small value of the energy gap $2 \Delta$ of typical superconductors, as compared to the energy of semiconductors, is the key factor in operation of superconductor single-photon detectors (SSPDs), efficient even at near-infrared telecommunication wavelengths (e.g., $1.3 \mu \mathrm{m}$ and $1.55 \mu \mathrm{m}$ ) [1]. Typically an SSPD consists of a meandering $\mathrm{NbN}$ superconducting stripe with a thickness of a few $\mathrm{nm}$ and a width of $\approx 100 \mathrm{~nm}$. The meander active area is $10 \times 10 \mu \mathrm{m}^{2}$ and it is incorporated 
into a $50 \Omega$ impedance coplanar-waveguide line. An incident optical photon, absorbed by the SSPD generates a very efficient and ultrafast avalanche of excited quasiparticles ( $\mathrm{QP}$ - broken Cooper pairs) at the site of photon absorption, resulting in a local resistive hotspot, i.e., the area of suppressed superconductivity. Expanding due to QP out-diffusion, the hotspot perturbs homogeneous flow of the biasing supercurrent, causing it redistribution around the hotspot towards the stripe edges ("side walks"). When the current in quasi-one-dimensional side walks exceeds the critical value, a phase slip center is created and an ultrafast voltage transient is generated across the device $[2,3]$.

Quantum efficiency (QE), defined as the ratio of the number of SSPD generated photoresponse transients to the incident number of photons per the device nominal area, rapidly decreases in SSPDs with the decrease in the photon energy and strongly depends on the stripe width and film thickness, as well as the NbN optical absorption [4].

This work demonstrates results of our modeling of the hotspot dynamics in $3.5 \mathrm{~nm}$ and $10 \mathrm{~nm}$ thick NbN SSPDs at $T=4.2 \mathrm{~K}$. Our approach accounts electric field penetration into the superconductor at the boundary of the resistive hotspot and explains the QE and hotspot diameter dependences on the incident photon energy, earlier experimentally observed [4].

\section{Experimental and numeric simulation results}

The coherence length of a superconductor $\xi$ defines the minimal size of the hotspot given by $S_{\mathrm{m}}=\pi(0.5 \xi)^{2} \approx 80 \mathrm{~nm}^{2}$ - for the NbN superconductor at $T=4.2 \mathrm{~K}$. Any smaller area will be tunneled through by the Cooper pairs giving no dissipation. The experimental QE vs. the incident photon energy dependence for a $3.5 \mathrm{~nm}$ and $10 \mathrm{~nm}$ thick SSPDs is presented in the inset of Fig. 1. As expected from the hotspot model, QE increases towards eventual saturation, corresponding to the film absorption. However, the data obtained using a $10 \mathrm{~nm}$ thick device show that for photon energies below approximately $1 \mathrm{eV}, \mathrm{QE}$ is practically zero, as the hotspot diameter appears to be either too small (smaller than $S_{\mathrm{m}}$ ), or insufficient to create a critical supercurrent redistribution and a phase slip center at the $0.9 I_{\mathrm{c}}$ perturbation current [3].

The concentration of excited QPs $C(r, t)$ as a function of the distance $r$ from the site of the photon absorption in an NbN superconducting film of the thickness $d$ is given by [2]:

$$
C(r, t)=\frac{M}{4 \pi D d t} \exp \left(-\frac{r^{2}}{4 D t}\right) \exp \left(-\frac{t}{\tau}\right)+C_{0},
$$

where $M, D$, and $C_{0}$ are the QP multiplication factor, diffusion coefficient, and equilibrium concentration, respectively. $\tau=\tau_{\mathrm{ep}}+\tau_{\mathrm{esc}} c_{\mathrm{e}} / c_{\mathrm{ph}}$ is the effective electron cooling time, with $\tau_{\text {ep }}$ corresponding to an electron-phonon interaction time, $\tau_{\text {esc }}$ phonon escape time, and $c_{\mathrm{e}}$ and $c_{\mathrm{ph}}$ specific heats of electrons and phonons, respectively. Our simulation results (see solid line plot in Fig. 1) show that within 


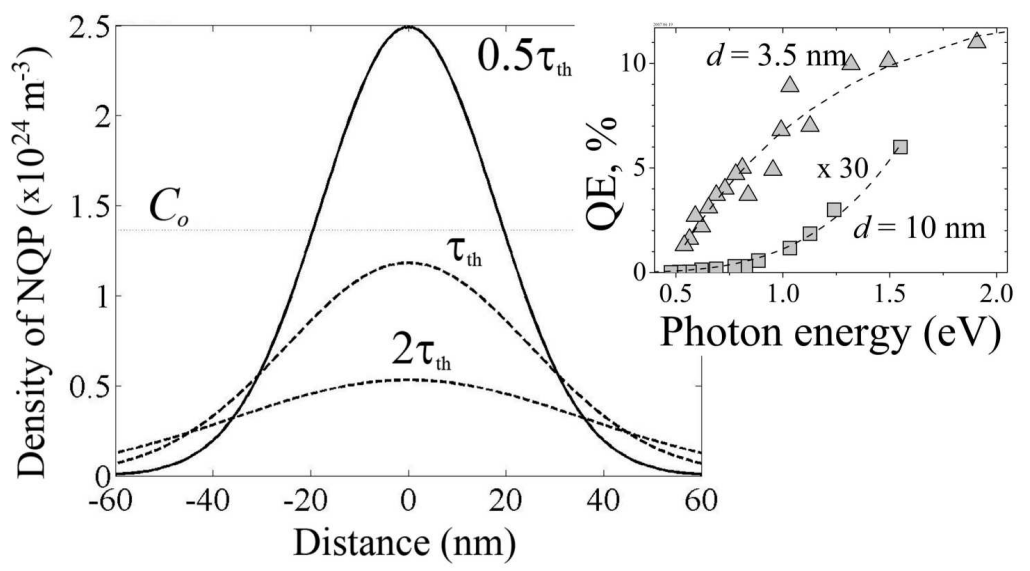

Fig. 1. The density of excited QP vs. the distance from the site of $1.55 \mu \mathrm{m}$ photon absorption for a $3.5 \mathrm{~nm}$ thick $\mathrm{NbN}$ film at $T=4.2 \mathrm{~K}$ with $C_{0}$ as the equilibrium concentration of $\mathrm{QP}$ and $\tau_{\mathrm{th}}$ as their thermalization time. Inset: the fit (dashed line) and experimental (symbols) dependences of QE vs. the incident photon energy for $3.5 \mathrm{~nm}(\boldsymbol{\Delta})$ and $10 \mathrm{~nm}(\mathbf{-})$ thick $100 \mathrm{~nm}$ wide NbN superconducting SSPDs, biased at $0.9 I_{\mathrm{c}}$.

time $t \sim 0.5 \tau_{\text {th }}$ (where $\tau_{\text {th }} \approx 7 \mathrm{ps}$ is the $\mathrm{QP}$ thermalization time in NbN at $T=4.2 \mathrm{~K}[5])$ the processes of $\mathrm{QP}$ thermalization and multiplication are in full progress in the NbN SSPD, the $C(r, t)$ exceeds $C_{0}$, and the developed hotspot area is $S \gg S_{\mathrm{m}}$.

Introducing $\xi=11.2 \mathrm{~nm}, D=4.5 \times 10^{-5} \mathrm{~m}^{2} / \mathrm{s}[2]$, and $\tau_{\text {ep }}=14 \mathrm{ps}[5]$, the initial time of the bias current redistribution at $T=4.2 \mathrm{~K}$ around the hotspot in our SSPD, $\tau_{\mathrm{jc}}$, given by $[6] \tau_{\mathrm{jc}}=\left[\tau_{\mathrm{ep}}^{-1}+\left(D / 2 \xi^{2}\right)\left(I / I_{\mathrm{c}}\right)^{2}\right]^{-1} \approx 5$ ps. Thus, the current redistribution in the $\mathrm{NbN}$ superconducting stripe starts already during the QP avalanche multiplication. This result allows us to predict that the hotspot development should be affected by the electric field penetration at the boundary between the hotspot and superconducting side walks [2]. The depth of electric field penetration, suppressing the superconducting gap in a superconductor, is given by $L_{E}=\sqrt{D \tau_{\mathrm{jc}}} \approx 13 \mathrm{~nm}$ at $T=4.2 \mathrm{~K}$. Assuming that QPs in the hotspot form a cloud of electrons confined by a cylinder defined by the hotspot model [2], the electric field penetration $L_{E}$ produces an outer ring of the "weakened" superconductor (see inset in Fig. 2) and forces the macroscopic supercurrent redistribution. The above approach gave us a very good agreement with the experimental data as is shown in Fig. 2 (dashed lines vs. symbols). When the condition $J_{\mathrm{sw}} \geq J_{\mathrm{c}}$ is satisfied, then the hotspot spreads across whole superconducting stripe and SSPD generates an ultrafast voltage transient with almost a negligible timing jitter.

We have presented a hotspot formation model for the single-photon detection mechanism in 2-dimensional superconducting NbN stripes biased with subcritical 


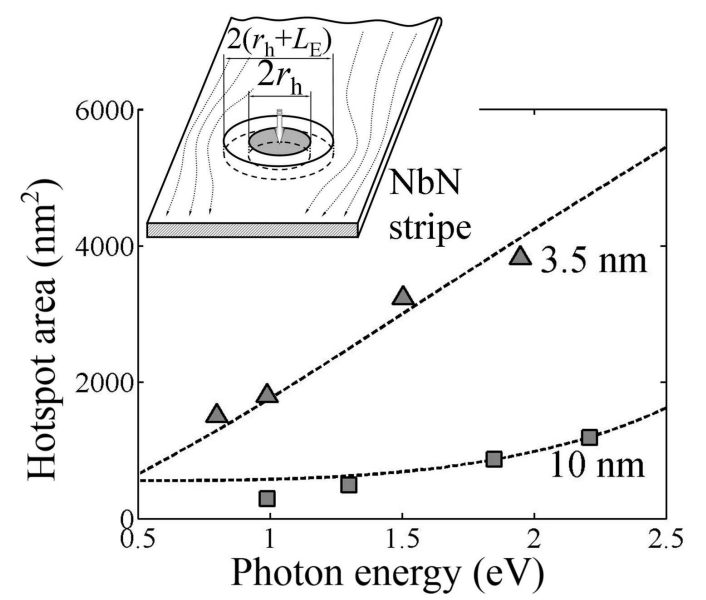

Fig. 2. The experimental data (symbols) and the fit (dashed lines) to area of hotspot produced by single photon of various quantum energy in $120 \mathrm{~nm}$ wide and $3.5 \mathrm{~nm}(\boldsymbol{\Lambda})$ and $10 \mathrm{~nm}(\mathbf{-})$ thick $\mathrm{NbN}$ films at $T=4.2 \mathrm{~K}$. Inset: A proposed model of the hotspot consisting of rh-radius hardcore (nonsuperconducting) and LE-radius softcore (weak superconductor) material.

currents. Our simulation results agree very well with the experiments and demonstrate that photon absorption together with the joint action of the electric field of the bias current result in a photoresponse $3.5 \mathrm{~nm}$ and $10 \mathrm{~nm}$ thick NbN SSPDs biased at $0.9 I_{\mathrm{c}}$ and maintained at temperature $T=4.2 \mathrm{~K} \ll T_{\mathrm{c}}$.

\section{Acknowledgments}

The work was supported in part by the U.S. AFOSR Grant FA9550-06-10348, with the additional support provided by the NYSTAR grant to the University of Rochester CAT-EIS Center.

\section{References}

[1] G.N. Gol'tsman, O. Okunev, G. Chulkova, A. Lipatov, A. Semenov, K. Smirnov, B. Voronov, A. Dzardanov, C. Williams, R. Sobolewski, Appl. Phys. Lett. 79, 705 (2001); A.D. Semenov, G.N. Gol'tsman, R. Sobolewski, Semicond. Sci. Technol. 15, R1 (2002).

[2] A.D. Semenov, G.N. Gol'tsman, A.A. Korneev, Physica C 351, 349 (2001).

[3] J. Zhang, W. Slysz, A. Verevkin, R. Sobolewski, O. Okunev, G.N. Gol'tsman, Phys. Rev. B 67, 132508 (2003).

[4] A. Verevkin, A. Pearlman, W. Stysz, J. Zhang, M. Currie, A. Korneev, G. Chulkova, O. Okunev, P. Kouminov, K. Smirnov, B. Voronov, G.N. Gol'tsman, R. Sobolewski, J. Mod. Opt. 51, 1447 (2004).

[5] K.S. Il'in, M. Lindgren, M. Currie, A.D. Semenov, G.N. Gol'tsman, R. Sobolewski, S.I. Cherednichenko, E.M. Gershenzon, Appl. Phys. Lett. 76, 2752 (2000).

[6] T.R. Lemberger, J. Clarke, Phys. Rev. B 23, 1100 (1981). 